\title{
The Refentse model for post-rape care: Strengthening sexual assault care and HIV post-exposure prophylaxis in a district hospital in rural South Africa
}

Julia C. Kim

Ian Askew

Population Council

Lufuno Muvhango

Ntabozuko Dwane

Tanya Abramsky

See next page for additional authors

Follow this and additional works at: https://knowledgecommons.popcouncil.org/departments_sbsr-rh

Part of the Domestic and Intimate Partner Violence Commons, Family, Life Course, and Society Commons, Health Policy Commons, International Public Health Commons, and the Women's Health Commons

How does access to this work benefit you? Let us know!

\section{Recommended Citation}

Kim, Julia C., Ian Askew, Lufuno Muvhango, Ntabozuko Dwane, Tanya Abramsky, Stephen Jan, Ennica Ntlemo, Jane Chege, and Charlotte Watts. 2009. "The Refentse model for post-rape care: Strengthening sexual assault care and HIV post-exposure prophylaxis in a district hospital in rural South Africa," FRONTIERS Final Report. Johannesburg: Population Council. 


\section{Authors}

Julia C. Kim, Ian Askew, Lufuno Muvhango, Ntabozuko Dwane, Tanya Abramsky, Stephen Jan, Ennica Ntlemo, Jane Chege, and Charlotte Watts 


\section{The Refentse Model for Post-Rape Care:}

\section{Strengthening Sexual Assault Care and HIV Post-Exposure Prophylaxis in a District Hospital in Rural South Africa}

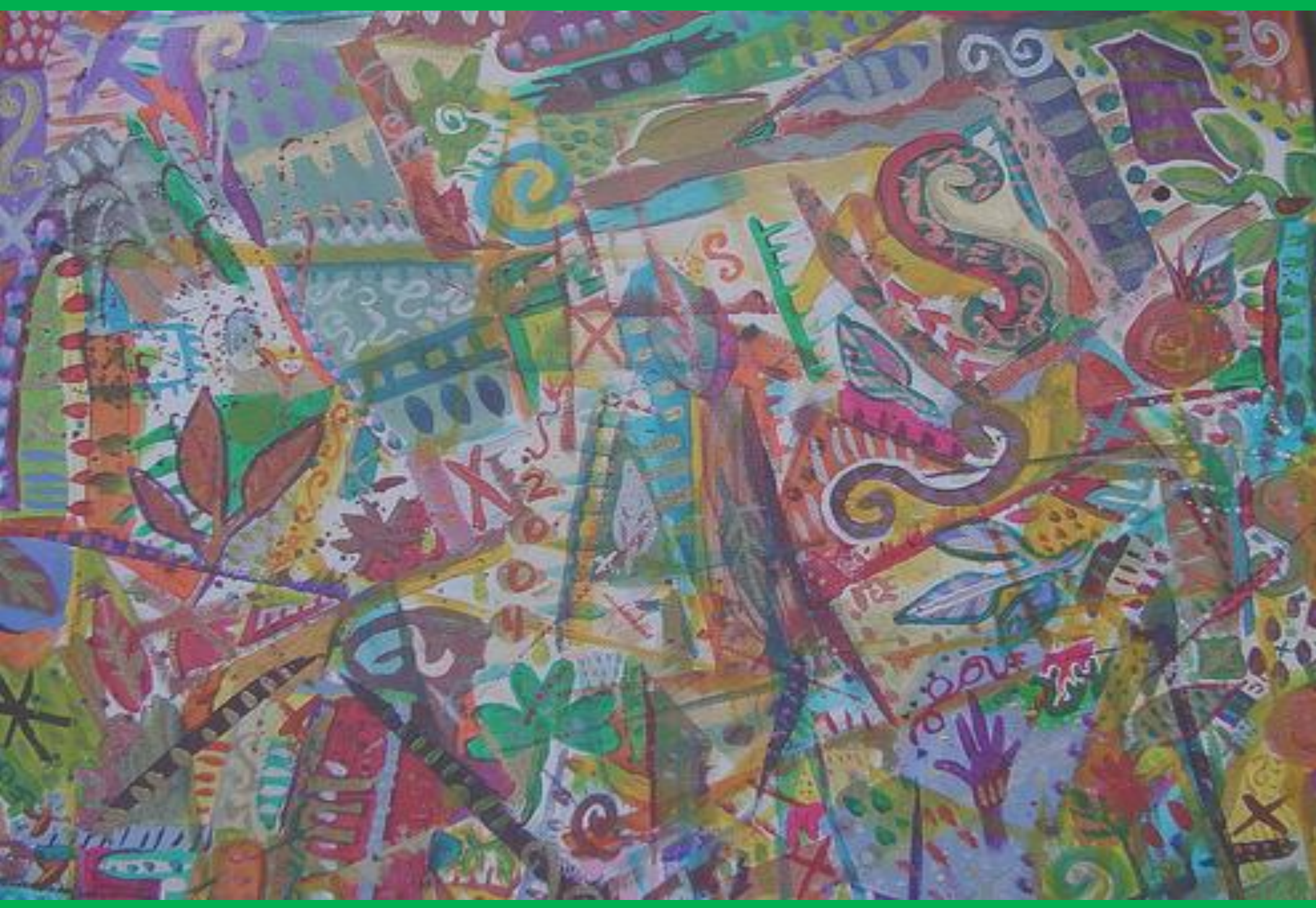




\section{(1) Population Council}

The Population Council conducts research worldwide to improve policies, programs, and products in three areas: HIV and AIDS; poverty, gender, and youth; and reproductive health.

(C) 2009 The Population Council, Inc.

This study was made possible by the generous support of the American people through the United States Agency for International Development (USAID) under the terms of Cooperative Agreement Number HRN-A-00-98-00012-00 and Subagreement numbers A104.25A and S107019A. The contents are the responsibility of the Population Council and do not necessarily reflect the views of USAID or the United States Government.

Any part of this publication may be reproduced without permission for limited distribution provided it is distributed without charge and the Population Council is acknowledged as its source. The Population Council would appreciate receiving a copy of any materials in which the text is used.

Suggested citation: Julia C. Kim, Ian Askew, Lufuno Muvhango, Ntabozuko Dwane, Tanya Abramsky, Stephen Jan, Ennica Ntlemo, Jane Chege and Charlotte Watts. 2009. The Refentse Model for Post-Rape Care: Strengthening Sexual Assault Care and HIV Post-Exposure Prophylaxis in a District Hospital in Rural South Africa. Population Council. 


\title{
The Refentse Model for Post-Rape Care: Strengthening Sexual Assault Care and HIV Post-Exposure Prophylaxis in a District Hospital in Rural South Africa
}

\author{
Julia C. Kim ${ }^{1,2}$ \\ Ian Askew ${ }^{3}$ \\ Lufuno Muvhango ${ }^{1}$ \\ Ntabozuko Dwane ${ }^{1}$ \\ Tanya Abramsky ${ }^{2}$ \\ Stephen Jan ${ }^{4}$ \\ Ennica Ntlemo ${ }^{1}$ \\ Jane Chege ${ }^{3}$ \\ Charlotte Watts ${ }^{2}$
}

\footnotetext{
Institutional affiliations at the time of the study:

${ }^{1}$ Rural AIDS and Development Action Research Programme (RADAR), School of Public Health, University of the Witwatersrand

${ }^{2}$ Gender, Violence and Health Centre, London School of Hygiene and Tropical Medicine

${ }^{3}$ Reproductive Health Program, Population Council

${ }^{4}$ The George Institute for International Health
}

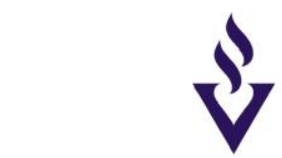

THE GEORGE INSTITUTE

for International Health
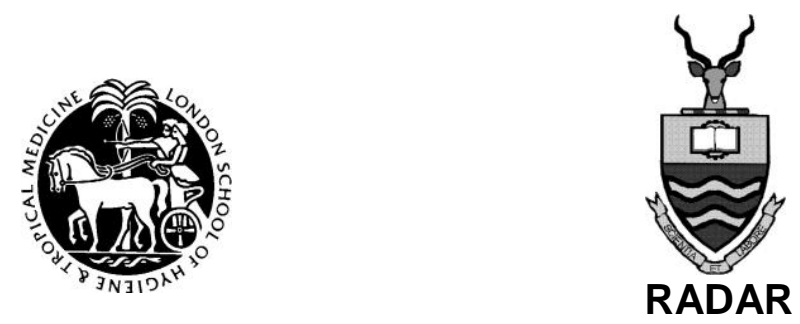


\section{ACKNOWLEDGEMENTS}

This study was made possible by the generous support of the American people through the United States Agency for International Development (USAID) under the terms of Cooperative Agreement Number HRN-A-00-98-00012-00 and Subagreement numbers A104.25A and S107019A. The contents are the responsibility of the authors and do not necessarily reflect the views of USAID or the United States Government.

The Refentse Study would not have been possible without the support and contributions of many people. Firstly, we would like to thank the members of the Project Advisory Committee for their guidance throughout the study. We would also like to acknowledge the Department of Health and Social Welfare in Limpopo Province and Mpumalanga Province for their encouragement and support. For their technical support in the training workshop and in developing intervention tools, we are grateful to the Western Cape Provincial Reference Group for Sexual Violence. Carol Wiebe's invaluable support to the project team is also gratefully acknowledged.

Finally, we are indebted to the many dedicated service providers at the study site and the courageous women and children who, as survivors of sexual assault, shared their valuable insights with us. 


\section{Table of Contents}

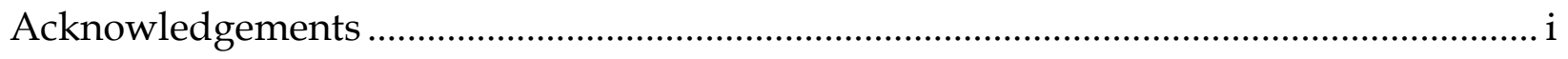

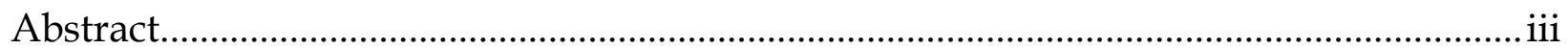

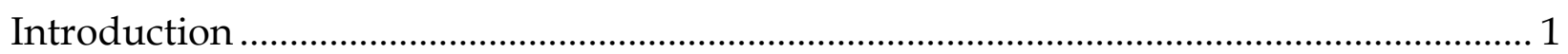

Comprehensive management of sexual assault: Changes and challenges ................ 1

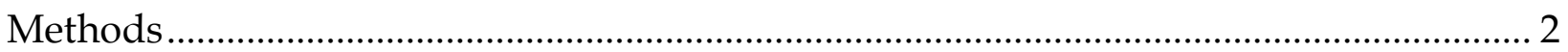

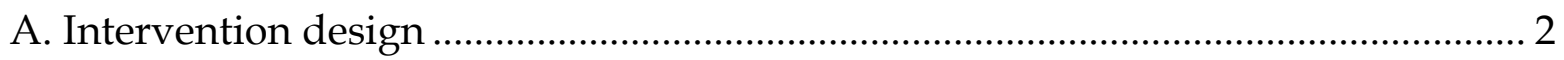

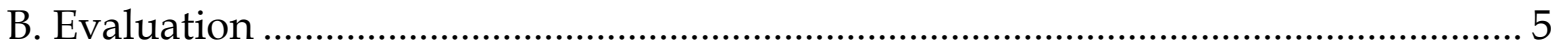

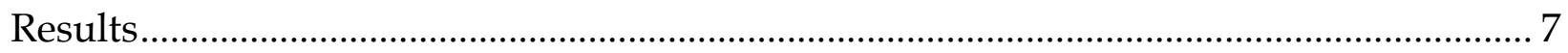

A. Changes in quality of post-rape care: Review of medical charts.......................... 7

B. Changes in patient-reported treatment completion and quality of care .............. 10

C. Role of Nurses in Delivering Post-Rape Care..................................................... 12

D. Intervention Costs .................................................................................................... 12

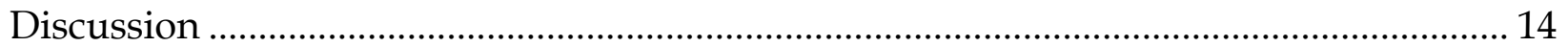

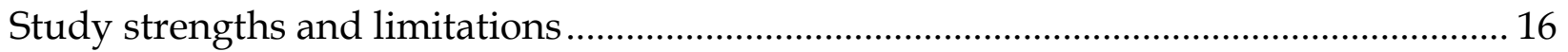

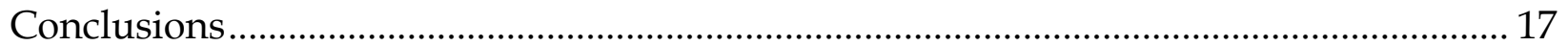

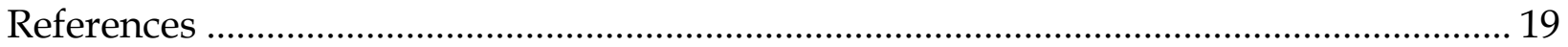




\section{ABSTRACT}

Objective: Sexual violence is widespread globally. HIV post-exposure prophylaxis (PEP) can be used following sexual assault to reduce the risk of HIV acquisition. Especially in settings such as South Africa, where both sexual violence and HIV are endemic, it is critical that health services provide timely and comprehensive care for sexual assault, including PEP. The Refentse study aimed to develop a nurse-driven, post-rape care model that could be integrated into existing reproductive health/HIV services within a rural South African hospital, and to evaluate the impact of this model on the quality of care delivered.

Methods: The study was based in a 450-bed district hospital and used a pre/post-intervention study design. After conducting formative research, a five-part intervention model was introduced, including: a sexual violence advisory committee; hospital rape management policy; training workshop for service providers; designated examining room; and community awareness campaigns. 334 hospital charts were reviewed to assess the quality of clinical care provided to patients, and interviews were conducted with 16 service providers and 109 patients. To provide an analysis of cost-consequences, incremental costs to the health sector of improving care through the intervention model were assessed.

Results: Initially, the service was fragmented, with multiple obstacles to the timely provision of care. Following the intervention, there were significant improvements in the quality of clinical history and examination, and the provision of pregnancy testing, emergency contraception, STI treatment; HIV counseling and testing, PEP, trauma counseling, and referrals. PEP completion rates increased from $20 \%$ to $58 \%$. The incremental cost of the intervention was US\$200 per case. When one-off development costs were excluded, the incremental cost per case was US\$58.

Conclusion: It is possible to improve sexual assault services including PEP within a rural South African hospital at modest cost, using existing staff and infrastructure. With additional training, nurses can play a central role in this care. 


\section{INTRODUCTION}

Sexual violence, both within and outside of conflict situations, is increasingly recognized as an important driver of the HIV epidemic within sub-Saharan Africa, where more than two-thirds of adults and nearly $90 \%$ of children infected with HIV reside. ${ }^{1-3}$ South Africa remains the country with the largest number of HIV infections in the world and in 2000, an estimated $40 \%$ of deaths in adults aged 15-49 were attributable to AIDS - making it the leading cause of death in the country. ${ }^{14}$ Simultaneously, South Africa has the highest worldwide annual incidence of rape reported to police. In spite of documented under-reporting, ${ }^{5}$ data indicate that in 2002 there were 52425 cases of rape (115 per 100000 population) reported to police in South Africa compared to, for example, 31.8 per 100000 in the United States or 8.5 per 100000 in Brazil ${ }^{6-8}$. Also alarming is the magnitude of infant and child rape, which has been the source of much media attention. Although accurate information on the prevalence of child sexual abuse is particularly difficult to obtain, South African police statistics for 1996-1999 indicated that for the crime of rape and attempted rape, 40\% of survivors were under the age of $18 .{ }^{9}$

Sexual violence can profoundly impact on the physical, emotional, mental and social well being of those who survive the assault. In addition to immediate genital and bodily injuries, patients are at risk of a range of medium and long-term health problems. These include sexually transmitted infections (STIs) including HIV, unwanted pregnancy, urinary tract infections, chronic pelvic pain, miscarriage, and mental health problems including depression, substance abuse, post-traumatic stress disorder, sleep difficulties and suicide. ${ }^{10}$ Many of these problems may persist for years after the assault, and adult survivors of child sexual abuse are known to be at greater long-term risk of substance abuse, mental health problems and unsafe sexual practices than the general population. ${ }^{211}$ 12

\section{Comprehensive management of sexual assault: Changes and challenges}

International guidelines have clearly laid out the central role of the health sector in providing comprehensive clinical care following sexual assault. ${ }^{13}$ However, a number of common challenges to service delivery have been noted in both industrialized and developing countries. These include: the absence of institutional policies or treatment protocols, lack of relevant training for healthcare workers; negative attitudes of service providers; fragmented and sub-standard provision of clinical care, lack of privacy, poor collection of forensic evidence, and lack of trauma counseling or psychosocial referrals. ${ }^{61014-22}$

In South Africa and internationally, the recent emergence of new preventive treatments such as HIV post-exposure prophylaxis (PEP) - the provision of anti-retroviral drugs to reduce the risk of HIV transmission following sexual assault - has heightened the importance of ensuring that health needs are met urgently. ${ }^{23}$ International guidelines recommend that PEP be given within 72 hours of exposure in order to maximize efficacy. ${ }^{13}$ Even in countries where PEP is not currently available, other important treatments such as emergency contraception (EC) to prevent pregnancy are also time-dependent. Where forensic evidence is collected for medico-legal evidence, the physical exam must be conducted as soon as possible in order to safeguard the quality of the evidence. Indeed the range of skills and services that must be coordinated in order to deliver expedient, effective care to the rape survivor necessitates an integrated, multi-sectoral delivery model that could prove enormously challenging to health systems, particularly in under-resourced settings. ${ }^{24}$ 
South Africa has made important headway in laying out a strong policy framework for addressing sexual violence through the health sector. In 2005 the Department of Health launched its National Sexual Assault Policy and Clinical Management Guidelines, which included a commitment to the national provision of $\mathrm{PEP}^{\mathrm{a}}$ following sexual assault. ${ }^{26}{ }^{27}$ Due to a range of obstacles and challenges, however, progress in developing policies has not been matched by implementation. Designated public service doctors (district surgeons) were traditionally responsible for treating rape cases. This system was fraught with problems, and in 1999 was phased out across the country. Although guided by a primary care approach and intended to ensure that sexual assault care could be delivered by non-specialist doctors, in practice, this shift left a sizeable capacity gap. The overwhelming majority of doctors now providing such care have had no specific training in managing sexual assault. ${ }^{62628}$ Compounded by the overall shortage of doctors, particularly in rural areas, the health sector has struggled to face the challenge of providing adequate health care for rape survivors. ${ }^{6142428-30}$

These challenges are not unique to South Africa, and are shared by many developing countries where both sexual violence and HIV/AIDS are major concerns. Thus, there is an urgent need to develop and test practical models for delivering comprehensive post-rape care within the constraints imposed by locally available resources. Given the shortage of doctors common to many settings, a key question concerns the feasibility of developing a service that is primarily nurse-driven.

Moreover, because PEP is one of a growing spectrum of inter-related reproductive health (RH) and HIV/AIDS interventions that healthcare workers are expected to implement, such models would ideally build on, rather than duplicate, existing health services. ${ }^{24} 31$ To date, few interventions have been rigorously evaluated, particularly in resource-poor settings. ${ }^{21}$ This paper presents results from an intervention study that evaluated a nurse-driven, comprehensive, post-rape care model, which was integrated into existing $\mathrm{HIV} / \mathrm{RH}$ services within a rural South African hospital.

\section{METHODS}

\section{A. Intervention design}

The Refentse study (meaning "we shall overcome" in Venda) was conducted from March 2003 to September 2007 and based at a 450-bed district hospital in rural South Africa. Formative research was used to conduct a baseline assessment of sexual assault services at the study hospital and to design the intervention model. Key informant interviews were conducted with 16 service providers (doctors, nurses, social workers, pharmacist, and police officers) to explore current problems with service delivery. In addition, 55 questionnaires were completed by service providers to document knowledge, attitudes and practices relating to the provision of post-rape care. Finally, a review of 144 medical charts documented objective evidence regarding the actual treatments undertaken for patients presenting to the hospital following sexual assault. Results of this formative research have been presented elsewhere. ${ }^{32}$ Key findings of importance to the intervention design included:

1) Capacity gaps: Few service providers had any prior training on post-rape management. Only $44 \%$ of doctors knew that PEP must be initiated within 72 hours, and among nurses and police this was even lower (15\% and 24\% respectively). Apart from social workers, few police (14\%) or

\footnotetext{
${ }^{a}$ South African PEP guidelines recommend the use of a two-drug regimen of zidovudine and lamivudine following sexual assault.
} 
healthcare workers (doctors $25 \%$, nurses $31 \%$ ) could identify the mandatory reporting age for cases of child abuse. Many healthcare workers and police held beliefs that implicitly blamed rape on a woman's behavior.

2) Institutional obstacles: Rape cases were not prioritized, but were directed to wait in the general Out Patients Department (OPD) queue. Hospital clerks routinely diverted patients to the police station if they had not yet opened a police report. Mapping the typical flow of rape cases from the police station through the hospital revealed a fragmented system, involving contact with up to 11 different providers, and many delays. There were numerous obstacles to accessing HIV voluntary counseling and testing (VCT) and PEP due to institutional and provider barriers, rather than patient delays in seeking care. These included the absence of VCT services afterhours (weekends and evenings), when most assault survivors presented to hospital. There was no clear pharmacy policy for dispensing PEP following sexual assault. During routine hospital hours, PEP was prescribed by doctors, and then collected by the patient from a pharmacist at the end of the hospital visit. After-hours PEP was supposed to be available through an on-call pharmacist, who would take calls from home. However, interviews with doctors indicated that this resulted in delayed access, both in cases of sexual assault, as well as needle-stick injuries among healthcare workers. In the absence of a PEP policy, the pharmacist was able to decide eligibility criteria based on his own opinions, which included the belief that women lie about rape. Finally, patients received little medication counseling, and because there was no formal system for referral of rape cases to social workers or other service providers, few received referrals for follow-up counseling after the assault.

3) Limited PEP delivery: As a result, among those patients who presented $<72$ hours of the assault (in time to receive PEP), about half were automatically excluded from PEP eligibility because VCT was unavailable at the time. Among those who did receive VCT and were eligible for PEP, the majority either received a starter pack (3 day regimen) or nothing at all. Of those offered starter packs, only 14\% returned again to receive additional medication. Taken together, among those who presented in time for PEP, and should have been eligible, only $16 \%$ ultimately received a full 28-day course of PEP.

Based on these findings, a five-part intervention model was developed and introduced in March 2005. This included establishing: 1) a sexual violence advisory committee (SVAC); 2) hospital rape management policy; 3) training workshop for service providers; 4) centralization and coordination of care through a designated examining room; and 5) community awareness campaigns (Figure 1). The intervention model relied entirely on existing hospital staff, with the exception of a research nurse, who provided in-service training to new nurses who joined the OPD following the initial workshop, and a SVAC Coordinator who convened quarterly meetings of the SVAC and liaised with its members between meetings. 


\section{Figure 1: Refentse intervention model for strengthening sexual assault care}

\section{1) Sexual Violence Advisory Committee}

To encourage buy-in and support from relevant stakeholders, a multi-sectoral Sexual Violence Advisory Committee (SVAC) was established, and included the hospital CEO, pharmacists, psychiatric nurse, HIV clinical nurses, doctors, OPD nursing management, social workers, and representatives from the local police station. The SVAC reviewed findings from the diagnostic phase in order to contribute to the design of the rest of the intervention model.

\section{2) Hospital Rape Management Policy}

The SVAC drafted a Hospital Rape Management Policy, which subsequently became an official hospital policy, signed by the CEO and relevant senior management. It was based on the National Management Guidelines for Sexual Assault and laid out treatment protocols that addressed specific problems identified during the diagnostic phase (e.g. 24-hour access to VCT, introduction of an early stat dose of PEP, and dispensing a full 28-day course of PEP on the initial visit)

\section{3) Training Workshop for healthcare workers and other providers}

A two-day training workshop for healthcare workers and other service providers was developed and implemented in March 2005. Participants were proposed by the SVAC, and included senior managers and healthcare workers from the hospital, a hospital pharmacist, district representatives from the Department of Health and Social Welfare, social workers, police, and a representative from the local Prosecutors Office. Drawing on training expertise from the Department of Maternal, Child, and Women's Health $(\mathrm{MCWH})$ in Western Cape Province, the workshop focused on developing a multisectoral approach to rape management, based on key competencies outlined in the National Management Guidelines. Key issues included addressing common myths and attitudes about rape, an overview of clinical care and PEP, exploring an expanded role for nurses (including forensic exam), and strengthening relationships between the health sector, social workers, police, and local prosecutors.

\section{4) Centralization and Co-ordination of Care through a Designated Examining Room}

Following the training workshop, police decided to prioritize PEP by bringing patients to hospital immediately - opening a docket only after clinical care had been received. Hospital clerks were also instructed not to redirect patients to the police station to open a case until they were treated. Senior Nursing Managers decided to centralize post-rape care through a designated room in the OPD, and to prioritize rape cases to bypass the OPD queue. With minor changes, the room was made more private, with all post-rape treatment (i.e. STI medication, pregnancy tests, emergency contraception, HIV tests, and PEP) being stocked and dispensed from a locked cupboard in the room. In addition, a set of clinical tools, designed to help health care workers to implement the National Management Guidelines for Sexual Assault were made available in the examination room.

\section{5) Community Awareness Campaigns}

To promote community awareness about sexual violence, PEP and availability of the post-rape services at the hospital and police station, the research nurse worked with local stakeholders (Department of Health and Social Welfare, police station, local schools and radio station) to distribute information pamphlets and convey key messages at community HIV awareness events. In addition, nurses at local PHC clinics were trained to include information regarding sexual violence, its health effects, and available services during their routine "morning health talks" for patients waiting in the clinic queues. 


\section{B. Evaluation}

The study utilized a pre/post intervention design to assess whether the intervention model could lead to improvements in: 1) the quality of general post-rape care (forensic history and exam, provision of EC, STI treatment, referrals); 2) the provision of PEP (access to VCT, provision of and completion of a full 28-day course); and 3) the efficiency and utilization of the service (number of service providers seen on first visit and volume of rape cases presenting to hospital per month).

Facility Inventory: A facility inventory was conducted at the study hospital at baseline and repeated 10 months after introducing the intervention, in order to document the availability of relevant tools and resources (e.g. rape management policies and protocols, private examining room, medications, laboratory tests, and forensic exam kits) and to document the coordination and roles of service providers. A facility checklist was developed based on national and international guidelines ${ }^{1327}$ and verified by key informant interviews with service providers, and a walk-through documentation of the site.

Hospital chart review: A structured chart review form was developed to assess the quality of clinical care provided to patients, and to describe the time of presentation, medications prescribed, investigations completed, referrals made, and thoroughness of the history and forensic examination. The form reflected standards set by the National DOH Guidelines on Management of Sexual Assault ${ }^{27}$ and was piloted prior to implementation. To minimize observer bias, specific criteria were established for assigning quantitative values to each indicator. For example, to assess the quality of the history and forensic exam, a scoring system was developed to document whether the healthcare worker had made note of a range of factors (e.g. evidence of injury) recommended in the National Guidelines and the Sexual Assault Evidence Collection Kit (SAECK). Clear criteria to determine whether the patient had been eligible for, and received, relevant diagnostic tests and treatment were also developed.

All cases of rape presenting to the hospital's Out-Patients' Department (OPD) that were recorded in the OPD Rape Register between March 1, 2003 and August 31, 2006 (42 months) were eligible for review. Inclusion criteria to the study comprised all cases of rape regardless of age, sex, or time of presentation. If a patient record could not be retrieved from medical records at first request, a second attempt was made to find it. If this failed, the record was excluded from the study (Figure 2).

Patient interviews: A structured patient interview was used to investigate quality of care from the rape survivor's perspective, using indicators corresponding to those in the chart review. Additional questions documented the nature of the incident, where patients first sought help, medication completion, uptake of referrals and counseling, as well as more subjective impressions of quality of care.

All cases of rape recorded in the OPD Rape Register between June 20, 2004 and August 31, 2006 (26 months, beginning after formative research period) were eligible for patient interview. At the end of the hospital visit, patients were informed about the study and offered a follow-up appointment in 4 weeks time. Because formative research indicated that return rates would likely be low due to economic constraints, a travel stipend was offered, to be paid upon return. Informed consent was sought to contact the patient/guardian by phone to remind them of the appointment one week beforehand. Face-to-face interviews were conducted in a private room with a female interviewer, using a structured questionnaire. All interviews were conducted in the local language. In the case of 
patients younger than age 14 years, interviews were conducted with the parent or guardian. All questions on medication completion used a calendar and a visual recognition tool, which contained unlabelled samples of each medication. Respondents were asked a series of questions: 1) was this medication given to you? 2) If so, what do you think it was for? 3) How did you take it (dose, interval and duration)? 3) How many pills remained when you stopped? The median time interval between initial presentation at the hospital and the patient interview was 34 days.

Data collection throughout the study was informed by international guidelines on technical, ethical, and safety considerations in conducting research on gender based violence. ${ }^{33} 34$ In addition, counseling and trauma debriefing were routinely offered to research staff as well as subjects. Ethical Clearance for the study was obtained from the University of the Witwatersrand Human Research Ethics Committee, the London School of Hygiene and Tropical Medicine Ethics Committee, and the Population Council IRB.

Data were entered into a Microsoft Access Database and analyzed using STATA 9.0. Crude risk ratios (RR) of the intervention effect on all of the outcome indicators were calculated along with 95\% confidence intervals. Risk ratios were estimated using Poisson regression models with robust standard errors. ${ }^{35}$ Multivariate Poisson regression was performed to calculate RRs adjusted for potential confounders including presentation $<72$ hours of the assault, presentation during hospital after-hours, age $<14$ years, the sex of the attending doctor, and whether the patient had been seen by a senior or junior doctor. The patient's sex was not included in the models because observations were almost exclusively female.

Economic analysis: The economic analysis aimed to document the additional costs to the health sector of improving post-rape care using the intervention model. These incremental costs included facility- and patient-level costs. ${ }^{\mathrm{b}}$ The facility-level costs of establishing and running the intervention were based on an analysis of financial statements and presented in terms of a cost per case. Capital costs were discounted at a 3\% annual rate. The incremental patient-level costs were estimated by determining the change in the levels of medication use, number of tests and referrals multiplied by their respective unit costs; which in turn were extracted from publicly available data sources (Table 4). The findings are presented in terms of cost per case by summing the facility- and patient-level components of cost. Because there is no single primary outcome for this study, the cost analysis enables the conduct of a cost consequences analysis whereby the costs of the intervention are simply set alongside a suite of outcome estimates. ${ }^{36}$

\footnotetext{
b "Patient-level costs" refer to the health sector costs of treating patients, as distinct from "patient costs", which refer to costs borne by patients themselves (e.g. transport). Because the focus of the costing study was to determine the additional cost to the health sector of implementing the intervention model, patient costs were not included in the analysis.
} 


\section{RESULTS}

\section{A. Changes in quality of post-rape care: Review of medical charts}

Among 409 rape cases recorded in the OPD Rape Register during the study period, 334 (81.7\%) of medical charts were successfully reviewed (Figure 2). Cases assessed in the pre- and postintervention phases were similar in terms of patient age, sex, time of presentation to hospital, proportion opening a police report, and eligibility criteria for relevant diagnostic tests and treatment (Table 1). In both groups, documented cases were almost exclusively female, had a mean age of 20 years (range 3 months to 94 years), and over one-quarter were children younger than 14 years old. The majority of cases presented within 72 hours of the assault and were eligible for both EC and PEP. The majority were attended to by a junior doctor (community service doctor, CSD) rather than a senior doctor.

Figure 2: Study enrolment

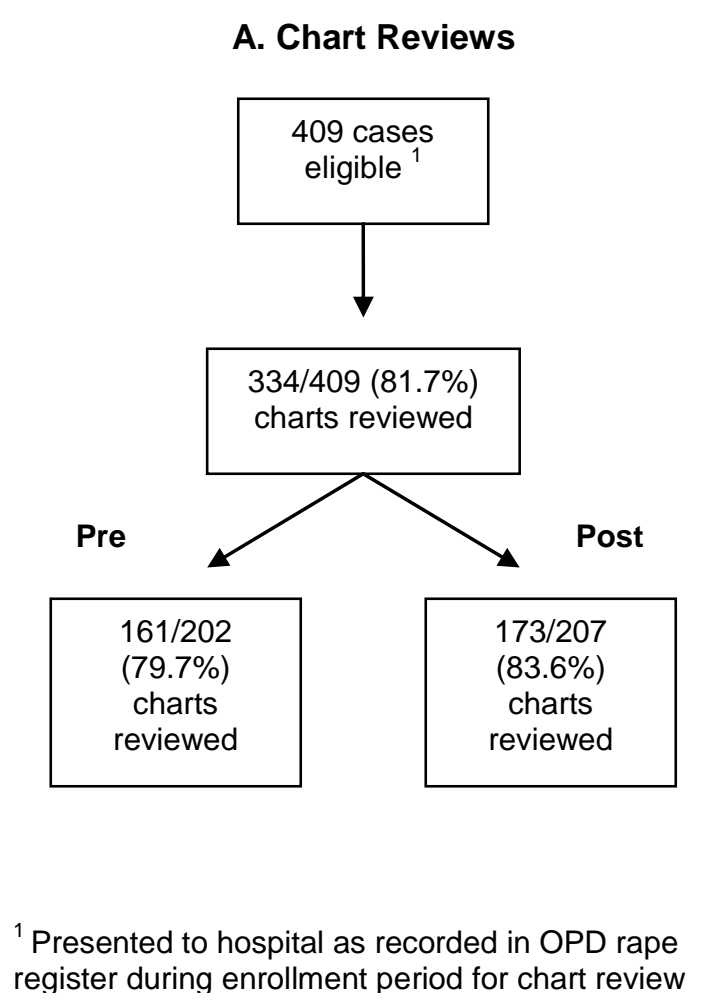

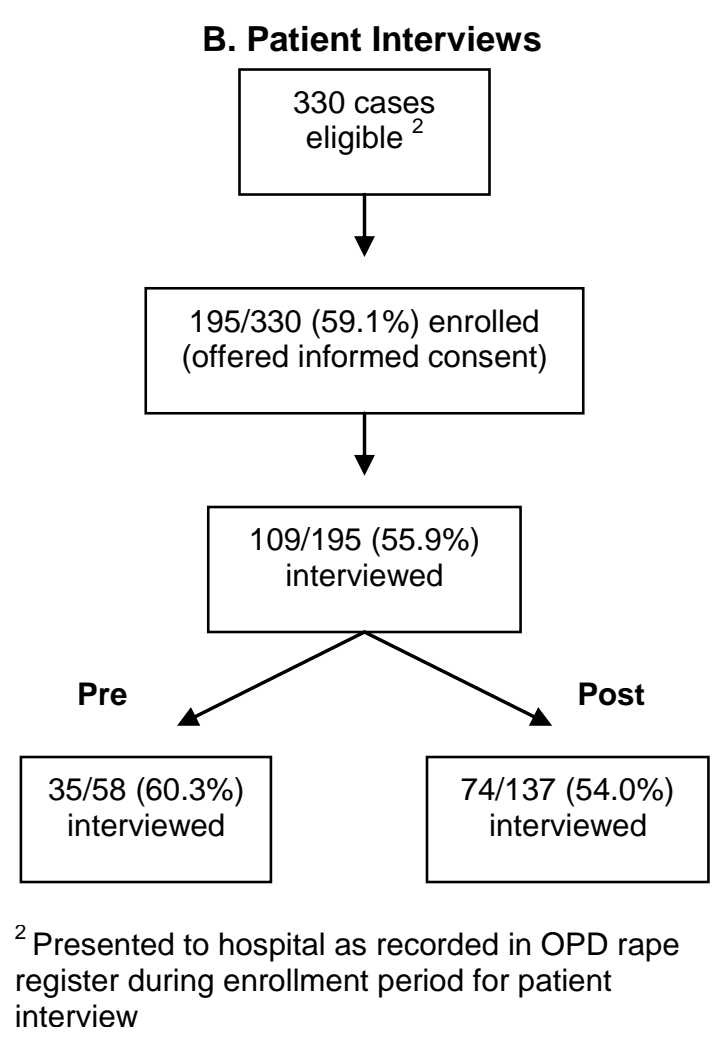


Table 1: Comparison of Pre vs. Post Intervention Groups (Chart Review) and Eligible vs. Interviewed Patients (Patient interview)

\begin{tabular}{|c|c|c|c|c|c|c|c|c|c|c|c|}
\hline \multirow{3}{*}{\multicolumn{2}{|c|}{ Characteristics }} & \multicolumn{5}{|c|}{ Chart Reviews } & \multicolumn{5}{|c|}{ Patient Interviews } \\
\hline & & \multicolumn{2}{|c|}{$\begin{array}{c}\text { Pre } \\
(\mathrm{N}=161)\end{array}$} & \multicolumn{2}{|c|}{$\begin{array}{c}\text { Post } \\
(\mathrm{N}=173)\end{array}$} & \multirow{4}{*}{$\begin{array}{c}\mathbf{P}^{*} \\
0.32^{\dagger}\end{array}$} & \multicolumn{2}{|c|}{$\begin{array}{l}\text { Eligible } \\
(N=195)\end{array}$} & \multicolumn{2}{|c|}{$\begin{array}{l}\text { Interviewed } \\
\quad(\mathrm{N}=109)\end{array}$} & \multirow[t]{2}{*}{$\mathbf{P}^{\star \star}$} \\
\hline & & $\mathrm{n} / \mathrm{N}$ & $\%$ & $\mathrm{n} / \mathrm{N}$ & $\%$ & & $\mathrm{n} / \mathrm{N}$ & $\%$ & $\mathrm{n} / \mathrm{N}$ & $\%$ & \\
\hline \multirow[t]{4}{*}{ Patient age } & Mean (SD) & \multicolumn{2}{|c|}{20.8 years $(12.3)$} & \multicolumn{2}{|c|}{19.4 years $(12.4)$} & & \multicolumn{2}{|c|}{$19.9(11.6)$} & \multicolumn{2}{|c|}{$18.9(11.8)$} & \multirow{2}{*}{$0.19^{\dagger}$} \\
\hline & range & \multicolumn{2}{|c|}{9 mos - 94.7 years } & \multicolumn{2}{|c|}{3 mos -68.6 years } & & \multicolumn{2}{|c|}{5 mos -68.6 years } & \multicolumn{2}{|c|}{5 mos -60.1 years } & \\
\hline & $<14$ years & $40 / 159$ & $25.2 \%$ & $51 / 166$ & $30.7 \%$ & 0.26 & $42 / 161$ & $26.1 \%$ & $31 / 92$ & $33.7 \%$ & 0.01 \\
\hline & $<18$ years & $74 / 159$ & $46.5 \%$ & $97 / 166$ & $58.4 \%$ & 0.03 & $88 / 161$ & $54.7 \%$ & $57 / 92$ & $62.0 \%$ & 0.03 \\
\hline \multicolumn{2}{|l|}{ Female } & $155 / 161$ & $96 \%$ & $172 / 173$ & $99 \%$ & 0.05 & $162 / 164$ & $99 \%$ & $91 / 93$ & $98 \%$ & 0.21 \\
\hline \multicolumn{2}{|c|}{ Presented $<72$ hours } & $121 / 141$ & $86 \%$ & $127 / 155$ & $82 \%$ & 0.37 & $121 / 146$ & $83 \%$ & $69 / 86$ & $80 \%$ & 0.31 \\
\hline \multicolumn{2}{|c|}{ Presented after hours } & $103 / 159$ & $65 \%$ & $109 / 170$ & $64 \%$ & 0.90 & $101 / 161$ & $63 \%$ & $59 / 92$ & $64 \%$ & 0.67 \\
\hline \multirow{2}{*}{$\begin{array}{l}\text { Examining } \\
\text { HCW }\end{array}$} & Senior doctor & $34 / 136$ & $25 \%$ & $68 / 171$ & $40 \%$ & 0.01 & $49 / 156$ & $31 \%$ & $28 / 92$ & $30 \%$ & 0.75 \\
\hline & Female & $73 / 135$ & $54 \%$ & $91 / 171$ & $53 \%$ & 0.88 & $83 / 154$ & $54 \%$ & $47 / 91$ & $52 \%$ & 0.50 \\
\hline \multicolumn{2}{|c|}{ Opened police report } & $134 / 147$ & $91 \%$ & $145 / 162$ & $90 \%$ & 0.63 & $143 / 154$ & $93 \%$ & $80 / 86$ & $93 \%$ & 0.93 \\
\hline \multicolumn{2}{|c|}{ Eligible for Pregnancy test } & $114 / 154$ & $74 \%$ & $126 / 172$ & $73 \%$ & 0.88 & $120 / 160$ & $75 \%$ & $64 / 92$ & $70 \%$ & 0.06 \\
\hline \multicolumn{2}{|c|}{ Preg test negative } & $31 / 36$ & $86 \%$ & $81 / 85$ & $95 \%$ & 0.08 & $71 / 77$ & $92 \%$ & $37 / 41$ & $90 \%$ & 0.49 \\
\hline \multicolumn{2}{|c|}{ Eligible for EC } & $109 / 114$ & $96 \%$ & $122 / 126$ & $97 \%$ & 0.62 & $114 / 120$ & $95 \%$ & $60 / 64$ & $94 \%$ & 0.50 \\
\hline \multicolumn{2}{|c|}{ Eligible for STI prevention } & $147 / 150$ & $98 \%$ & $167 / 168$ & $99 \%$ & 0.26 & $154 / 154$ & $100 \%$ & $91 / 91$ & $100 \%$ & 0.99 \\
\hline \multicolumn{2}{|c|}{ Eligible for VCT } & $119 / 140$ & $85 \%$ & $135 / 161$ & $84 \%$ & 0.78 & $128 / 151$ & $85 \%$ & $73 / 87$ & $84 \%$ & 0.73 \\
\hline \multicolumn{2}{|c|}{ HIV negative } & $72 / 90$ & $80 \%$ & $99 / 111$ & $89 \%$ & 0.07 & $104 / 120$ & $87 \%$ & $64 / 70$ & $91 \%$ & 0.07 \\
\hline \multicolumn{2}{|c|}{ Eligible for PEP } & $55 / 88$ & $63 \%$ & $77 / 110$ & $70 \%$ & 0.27 & $78 / 115$ & $68 \%$ & $46 / 66$ & $70 \%$ & 0.62 \\
\hline
\end{tabular}

${ }^{\dagger}$ t-test p-value

* Chi-2 test comparing characteristics of charts reviewed in the post-intervention period versus charts reviewed in the pre-intervention period

${ }^{* *}$ Chi-2 test comparing characteristics of those interviewed versus those eligible but not interviewed 
Comparing 11 pre- and post-intervention indicators of post-rape care from the chart review, after adjusting for the potentially confounding factors noted above, the quality of post-rape care improved significantly across all indicators (Table 2), including the quality of clinical history, physical exam, provision of pregnancy testing and EC. The provision of VCT increased substantially from $60 \%$ to $87 \%$, while syndromic treatment of STIs increased only slightly from $88 \%$ to $92 \%$.

Significant improvements were also seen in provision of PEP. After the intervention, patients were $57 \%$ more likely to have received any PEP.- whether a starter pack or full 28 day course. Those presenting in the post-intervention period were more than three times as likely to receive the full course on their first visit compared to the $15 \%$ of pre-intervention patients who had received this. Significant improvements were also seen in prescription of anti-emetics and referrals to other service providers (e.g. social worker, or psychiatric nurse) for counseling.

Table 2: Chart Review: Changes in Quality of Sexual Assault Care Provided Before and After Intervention

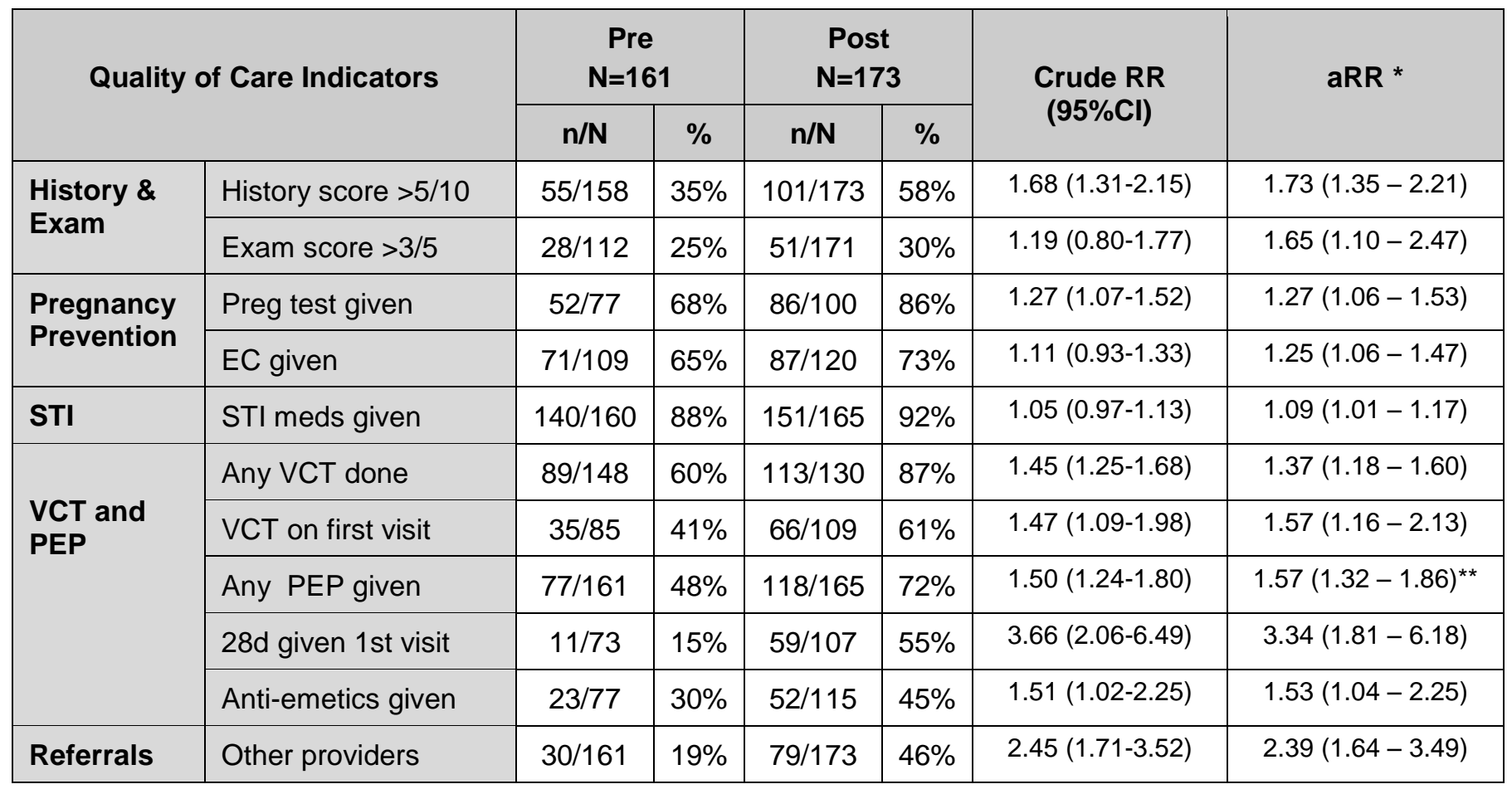

* aRRs adjusted for whether or not presented in $<72 \mathrm{hrs}$, whether age $<14$, whether seen by senior doctor, sex of healthcare worker, whether presented to hospital after-hours.

** additionally adjusted for HIV status 


\section{B. Changes in patient-reported treatment completion and quality of}

care

Among the 330 cases recorded in the OPD register during the interview period, 195 (59.1\%) were enrolled into the study, and of these, $109(55.9 \%)$ were successfully interviewed (Figure 2). Table 1 describes the characteristics of those patients from whom informed consent was sought, compared to those who were successfully interviewed. The two populations were very similar across most indicators. Those interviewed were also similar to the overall group in whom medical charts were reviewed.

Comparing patients interviewed in the pre- and post-intervention periods, improvements were noted in the areas of pregnancy counseling, PEP provision, and perceptions of the hospital service. (Table 3). After the intervention, patients were $29 \%$ more likely to report that they had been counseled about their risk of pregnancy, and there were significant improvements in most indicators relating to PEP. Patients were 35\% more likely to recognize PEP medications, and the proportion who understood that their purpose was to prevent HIV infection also increased significantly. Mirroring changes seen in the chart review, patients were more likely to report having received the full 28 day course of PEP on their first visit. In terms of PEP completion, they were more than three times as likely to report that they had taken a full 28-day course of PEP. The time interval between the assault and taking the first dose of PEP also decreased from 28 hours to 18 hours. The proportion of patients reporting any side effects while taking PEP (around 43\%) remained the same.

Based on the hospital rape register, utilization of services increased after the intervention, with the mean number of rape cases presenting to hospital increasing from 8 to 13 cases per month (RR 1.67; 95\% CI 1.37-2.03). Fewer patients were diverted to the police before being treated, and the proportion of patients having to see six or more service providers on their first visit decreased from 86\% to 54\% (Table 3). However, these improvements did not translate into a decrease in the overall time spent at hospital, with similar proportions reportedly staying for more than four hours. As previously reflected in the chart review, those interviewed after the intervention were more than twice as likely to report having received an appointment for follow-up counseling with another service provider. Patients' perceptions of the care they had received at the hospital improved substantially. These included improvements in their subjective ratings of health care providers' attitudes, the privacy of the forensic exam, and their perceptions of whether any counseling received had been helpful. In contrast, there were no significant changes seen in patient's impressions of, or confidence in the police and judicial system. 
Table 3: Patient - Reported Treatment Completion and Quality of Care

\begin{tabular}{|c|c|c|c|c|c|c|c|}
\hline \multicolumn{2}{|c|}{ Patient - Reported Indicators } & \multicolumn{2}{|c|}{$\begin{array}{c}\text { Pre } \\
\mathrm{N}=35\end{array}$} & \multicolumn{2}{|c|}{$\begin{array}{l}\text { Post } \\
\mathrm{N}=74\end{array}$} & Crude RR & $\mathrm{aRR}^{*}$ \\
\hline \multirow{4}{*}{$\begin{array}{l}\text { Pregnancy } \\
\text { prevention }\end{array}$} & Told about preg risk & 20/32 & $63 \%$ & $50 / 62$ & $81 \%$ & $1.29(0.96-1.74)$ & $1.29(0.97-1.74)$ \\
\hline & Recognized Ovral & $11 / 25$ & $44 \%$ & $31 / 57$ & $55 \%$ & $1.24(0.75-2.05)$ & $1.23(0.76-1.98)$ \\
\hline & Knew purpose & $5 / 11$ & $46 \%$ & $22 / 31$ & $71 \%$ & $1.56(0.78-3.12)$ & $1.64(0.82-3.28)$ \\
\hline & Took both doses & $7 / 8$ & $88 \%$ & $26 / 31$ & $84 \%$ & $0.96(0.70-1.30)$ & $0.91(0.70-1.18)$ \\
\hline \multirow{4}{*}{$\begin{array}{l}\text { STI } \\
\text { prevention }\end{array}$} & Recognized Doxycycline & $18 / 25$ & $72 \%$ & $37 / 57$ & $65 \%$ & $0.90(0.66-1.23)$ & $0.90(0.66-1.23)$ \\
\hline & Knew purpose & $1 / 18$ & $6 \%$ & $7 / 37$ & $19 \%$ & $3.41(0.44-26.11)$ & $3.41(0.41-28.45)$ \\
\hline & Correct dosing & $9 / 18$ & $50 \%$ & $25 / 37$ & $68 \%$ & $1.35(0.81-2.27)$ & $1.36(0.82-2.23)$ \\
\hline & Took $\times 7$ days & $4 / 14$ & $29 \%$ & $12 / 29$ & $41 \%$ & $1.45(0.56-3.73)$ & $1.50(0.54-4.15)$ \\
\hline \multirow{7}{*}{ PEP } & Recognized PEP & $18 / 33$ & $55 \%$ & $51 / 68$ & $75 \%$ & $1.38(0.98-1.94)$ & $1.35(0.96-1.90)$ \\
\hline & Knew purpose & $2 / 16$ & $13 \%$ & $24 / 49$ & $49 \%$ & $3.92(1.03-14.93)$ & $3.93(1.14-13.56)$ \\
\hline & Correct dosing & $10 / 15$ & $67 \%$ & $43 / 50$ & $86 \%$ & $1.29(0.88-1.88)$ & $1.31(0.91-1.89)$ \\
\hline & Full course given $1^{\text {st }}$ visit & $14 / 36$ & $39 \%$ & $15 / 26$ & $58 \%$ & $1.48(0.87-2.52)$ & $1.46(0.86-2.47)$ \\
\hline & Took $\times 28$ days & $3 / 15$ & $20 \%$ & $21 / 36$ & $58 \%$ & $2.92(1.01-8.41)$ & $3.13(1.10-8.93)$ \\
\hline & PEP Side effects & $8 / 17$ & $47 \%$ & $24 / 57$ & $42 \%$ & $0.89(0.49-1.62)$ & $0.94(0.54-1.64)$ \\
\hline & Hours between assault and $1^{\text {st }}$ dose & \multicolumn{2}{|c|}{$27.9(33.1)$} & \multicolumn{2}{|c|}{$18.1(14.4)$} & $-9.8(-21.4-1.9)^{2}$ & $-9.71(-21.31-1.89)^{2}$ \\
\hline \multirow{4}{*}{$\begin{array}{l}\text { Efficiency of } \\
\text { Service }\end{array}$} & Redirected from hospital to police & $7 / 16$ & $44 \%$ & $6 / 35$ & $17 \%$ & $0.39(0.13-1.17)$ & $0.34(0.11-1.04)$ \\
\hline & Saw 6 or more providers & $30 / 35$ & $86 \%$ & $40 / 74$ & $54 \%$ & $0 . .63(0.39-1.01)$ & $0.64(0.40-1.02)$ \\
\hline & Spent $>4$ hours at hospital & $14 / 35$ & $40 \%$ & $36 / 74$ & $49 \%$ & $1.22(0.66-2.25)$ & $1.23(0.66-2.28)$ \\
\hline & Received referral & $7 / 34$ & $21 \%$ & $36 / 73$ & $49 \%$ & $2.40(1.19-4.84)$ & $2.33(1.14-4.77)$ \\
\hline \multirow{3}{*}{$\begin{array}{l}\text { Perceptions } \\
\text { of Hospital }\end{array}$} & HCW attitude good/excel & $25 / 33$ & $76 \%$ & $69 / 72$ & $96 \%$ & $1.27(1.04-1.54)$ & $1.26(1.04-1.54)$ \\
\hline & Forensic exam private & $12 / 34$ & $36 \%$ & $70 / 72$ & $97 \%$ & $2.75(1.74-4.36)$ & $2.73(1.73-4.32)$ \\
\hline & Counseling was helpful & $16 / 26$ & $62 \%$ & $64 / 65$ & $99 \%$ & $1.60(1.18-2.18)$ & $1.59(1.17-2.16)$ \\
\hline \multirow{3}{*}{$\begin{array}{l}\text { Perceptions } \\
\text { of Police }\end{array}$} & Police attitude good/excel & $18 / 34$ & $53 \%$ & $42 / 69$ & $61 \%$ & $1.15(0.79-1.67)$ & $1.15(0.79-1.68)$ \\
\hline & Think > half cases go to trial & $10 / 27$ & $37 \%$ & $13 / 48$ & $27 \%$ & $0.73(0.37-1.44)$ & $0.76(0.38-1.52)$ \\
\hline & Confident own case will go to trial & $8 / 22$ & $36 \%$ & $11 / 50$ & $22 \%$ & $0.61(0.28-1.30)$ & $0.59(0.28-1.25)$ \\
\hline
\end{tabular}

$*$ aRRs adjusted for whether age $<14$ except where already stratified by age. Additional variables were not included in the analysis as they did not remove the effect of the intervention and given limited sample sizes, did not have the power to estimate further adjusted results with any precision.

${ }^{1}$ Difference in number of days took treatment, post- versus pre-intervention

${ }^{2}$ Difference in hours until treatment, post- versus pre-intervention 


\section{Role of Nurses in Delivering Post-Rape Care}

The facility inventory and key informant interviews indicated that at baseline, most care was delivered by a doctor, with the nursing role confined primarily to obtaining the medical chart, taking vital signs, and assisting the doctor. Following the intervention, this role had expanded to include documenting the rape history, providing acute trauma debriefing, providing a stat dose of PEP, and taking a pregnancy test. If VCT-certified, the nurse would also perform VCT. Using the hospital protocol (developed as part of this intervention), nurses then assembled the treatment package (STI medications, EC and PEP), provided medication counseling, and made referrals to the social worker or psychiatric nurse. However, few nurses reported conducting a forensic examination following the intervention, and the chart review indicated that $95 \%$ of exams were still conducted by a doctor.

\section{Intervention Costs}

The estimated additional cost for delivering the intervention was $\$ 200($ ZAR1,406) per case. This included the facility-level costs associated with capital investment, training and development, the salaries of the Research Nurse and SVAC Coordinator and the patient-level costs associated with medication use, tests and referrals. Once the one-off materials development costs and initial costs of the Research Nurse and SVAC Coordinator were excluded, the incremental intervention costs decreased to $\$ 58$ (ZAR 408) per case. 
Table 4: Incremental costs of Intervention

\begin{tabular}{|c|c|c|}
\hline Item & $\begin{array}{c}\text { Cost } 2006^{1} \\
\text { ZAR }\end{array}$ & US\$ \\
\hline \multicolumn{3}{|l|}{ Capital } \\
\hline Computers & 10,000 & 1,429 \\
\hline Printer/fax & 3,000 & 429 \\
\hline Office furniture & 3,275 & 468 \\
\hline Training and development of materials & 190,231 & 27,176 \\
\hline Office rent & 21,780 & 3,111 \\
\hline \multicolumn{3}{|l|}{ Personnel } \\
\hline SVAC $^{2}$ Coordinator & 72,600 & 10,371 \\
\hline Research Nurse & 127,050 & 18,150 \\
\hline \multicolumn{3}{|l|}{ Consumables and overheads } \\
\hline Local transport & 38,115 & 5,445 \\
\hline SVAC $^{2}$ meetings & 3,480 & 497 \\
\hline Phone/email/fax & 43,560 & 6,223 \\
\hline Stationery and photocopying & 43,560 & 6,223 \\
\hline Total facility-level costs & 556,651 & 79,522 \\
\hline Facility-level cost per case (409 cases) & 1,361 & 194 \\
\hline $\begin{array}{l}\text { Incremental patient-level costs (average) }^{3} \\
\text { ARV one month } \\
\text { Emergency contraception } \\
\text { Emt } \\
\text { STI (doxycycline treatment) } \\
\text { Pregnancy test } \\
\text { Anti-emetics }^{8} \\
\text { Referral to other medical service }^{y}\end{array}$ & $\begin{array}{c}38.26 \\
0.33 \\
0.55 \\
1.84 \\
0.16 \\
4.23 \\
\end{array}$ & $\begin{array}{l}5.47 \\
0.05 \\
0.08 \\
0.26 \\
0.02 \\
0.60 \\
\end{array}$ \\
\hline Subtotal of incremental patient-level costs & 45.36 & 6.48 \\
\hline Total incremental cost per case ${ }^{10}$ & 1406 & 200 \\
\hline Total incremental cost per case beyond initial development phase ${ }^{11}$ & 408 & 58 \\
\hline
\end{tabular}

${ }^{1}$ Exchange rate US $\$ 1=7$ ZAR

2 SVAC $=$ Sexual Violence Advisory Committee

${ }^{3}$ Estimated by subtracting pre-intervention cost from the post-intervention cost of each item. Pre- intervention cost is the unit cost multiplied by the pre-intervention risk for that item. Post intervention cost is pre-intervention cost multiplied by the adjusted risk ratio for that item e.g. to estimate cost of one month ARV (based on a unit cost of ZAR 109) $=109 \times 0.15 \times 3.34-109 \times 0.15=38.26$.

${ }^{4}$ Unit cost of ZAR 109 for a 1 month course of AZT and 3TC. ${ }^{58}$

${ }^{5}$ Unit price of ZAR 2 for total of Ovral 59

${ }^{6}$ Unit price of ZAR 7 for 7 days of Doxycycline and stat doses of Ciprofloxacin and Metronidazole ${ }^{60}$

${ }^{7}$ Assumed unit cost ZAR10 per test

${ }^{8}$ Unit cost for 1 pack of 10 Metoclopramide of ZAR 1 based on the 2002 Pharmaceutical Price List http://www.doh.gov.za/programmes/index.html (accessed 16 January 2008)

${ }^{9}$ Unit price of ZAR 16 for allied health worker consultation based on the Uniform Fee Schedule http://www.doh.gov.za/programmes/upfs-f.html (accessed 16 January 2008)

${ }^{10}$ Program cost per case plus subtotal of average patient costs

${ }^{11}$ Estimate of intervention costs after primarily one-off development costs are excluded. 


\section{DISCUSSION}

To our knowledge, this is the first intervention study from an African setting that has attempted to evaluate a comprehensive model for strengthening the health sector response to sexual violence. The capacity gaps and institutional barriers identified in the formative research were not unique to our setting and reflect those that have been previously reported in South Africa and internationally. ${ }^{61014}$ ${ }^{21}$ Following the introduction of the intervention model, both the chart review and patient interviews indicated substantial improvements across many domains, including the quality of history and exam and the provision of pregnancy testing, EC, STI treatment; VCT and PEP, as well as follow-up counseling and referrals. In addition, patient interviews indicated that the service had become more private, streamlined, and patient-centered.

Given the generalized HIV epidemic in South Africa, it is not surprising that interviews with rape survivors indicated that fear of HIV infection was their greatest concern. Our study found high acceptance rates for VCT and PEP, once institutional obstacles to accessing the services were removed. High PEP acceptance rates following sexual assault have previously been reported in South Africa and other high HIV-prevalence countries, ${ }^{37-41}$ although they vary widely in other settings where the actual and perceived risk of HIV infection may be much lower. ${ }^{23}{ }^{42-44}$ In our study, completion rates for a 28 -day PEP regimen increased to $58 \%$ following the intervention, which is similar to the figure of $57 \%$ reported at a specialized urban rape crisis center in South Africa ${ }^{40}$ and higher than rates that have been reported in North America. ${ }^{23}{ }^{434}$ However, this should be interpreted with caution as PEP completion and adherence rates are difficult to compare and vary widely across studies and settings, due to different eligibility criteria for initiating PEP, variations in drug regimens, and different approaches to defining and measuring adherence. $.^{30} 4245-47$. However, as with PEP acceptance rates, it is also possible that local population-level HIV prevalence rates and self-perceived risk of infection may play a key role in motivating treatment adherence, thus contributing to the variation in completion rates seen between sites. There is some evidence to suggest this may be the case. ${ }^{24} 4248$

Baseline findings indicated that, in our population, few rape survivors were willing or able to return for follow-up visits - a finding not confined to remote or resource-poor settings. ${ }^{232542-4449}$ The intervention model introduced a policy for prescribing the full 28 day regimen on the initial hospital visit for those who were eligible for PEP. A potential concern is that providing a full regimen without further counseling visits might lead to poor adherence and a waste of expensive medication. However, patients who are unable to return to hospital and only complete a starter pack of PEP also receive incomplete treatment, and so these pills too are effectively "wasted". When we compared those given a full course of PEP on the first visit to those given a starter pack with follow-up appointments, the former group were much more likely to have taken PEP for 28 days $71 \%$ vs. $29 \%$ ). Thus, following this intervention, patients were more likely to report having received any PEP, to have received a full 28-day course on their first visit, and to have completed the full 28-day regimen. South African guidelines make a provision for dispensing the full PEP regimen on the initial visit for those eligible patients who are unable to return due to economic or logistical reasons. ${ }^{27}$ Our findings lend support to the feasibility and practicality of this recommendation. Moreover, to support PEP adherence, national and international guidelines highlight the importance of providing adequate medication counseling, alongside anti-emetics for control of nausea (a common side-effect), particularly given the multiple PEP, STI, and EC medications prescribed. ${ }^{1327}$ In our study, the provision of anti-emetics increased after the intervention, as did the proportion of patients who could report the correct dosing interval and purpose of PEP, suggesting that these 
elements may have contributed to the improvements in PEP completion observed. Interventions to maximize adherence and minimize subsequent HIV exposures are critical to enhancing the potential effectiveness of PEP, an area where further work is needed. ${ }^{25} 50$

Institutional delays in initiating the first dose of PEP have been widely reported in South Africa. ${ }^{28}{ }^{21}$ Following the training intervention, police prioritized PEP by bringing patients to hospital before opening a police report. The hospital rape management policy also introduced a "stat" dose of PEP at the start of the treatment protocol. These steps appear to have contributed to a reduction in the mean time to receiving the first dose of PEP from 28 hours to 18 hours after the assault - an important outcome as numerous animal studies suggest greater efficacy of PEP the sooner it is taken after exposure. ${ }^{135253}$

The study also shows that it is possible to substantially expand the role of nurses in the management of sexual assault. Following the intervention, nurses took the lead in implementing the post-rape care treatment protocol, a significant change from the previously doctor-driven service. However, a disappointing finding from the study, was the lack of impact on building nurses' capacity and willingness to perform the forensic examination. In retrospect, this reflects a number of factors. Firstly, it is likely we under-estimated the intensity of training required to develop proficiency and confidence in this area. The study employed a designated research nurse who received three weeks of training and mentorship under the guidance of experts from the Department of Health in the Western Cape, followed by ongoing supervision by doctors at the study hospital. Although she eventually gained the confidence and skill to conduct forensic exams, she was unable to transfer these skills to other nurses in OPD. KI interviews reveal that nurses were in general reluctant to learn about this aspect of post-rape management, and were intimidated by the long time required to conduct the forensic exam and the prospect of presenting evidence in court. Moreover, many felt there was a lack of clarity in current government policies as to whether nurses' expert testimony would be admissible in court. These barriers in the deployment of forensic nurses have been reported previously in South Africa and elsewhere. ${ }^{6142654}$ Although sexual assault nurse examiners have been found to be proficient in collecting forensic evidence in the United States, ${ }^{556}$ in South Africa, both the training approaches, and the development of norms and standards for forensic nurses are still evolving. This is clearly an area where further experience and research is needed.

The majority of resources used in the intervention model were facility based and were fully costed in the analysis. In other settings where such a program might build onto existing hospital infrastructure, costs are likely to be lower than those reported here. Moreover, it is likely that many of the significant start-up costs associated with developing the intervention were one-off and that the initial salary costs of the Research Nurse and SVAC Coordinator would not be incurred at a later stage once established routines became institutionalized. When these savings are taken into account the cost of the intervention reduces to $\$ 58$ per case. In the context of an estimated lifetime cost of ART in South Africa of approximately $\$ 6,700{ }^{57}$ it can be argued that this intervention represents a minimal but important investment of resources. 


\section{STUDY STRENGTHS AND LIMITATIONS}

A recent review of health-care based interventions for sexual violence found few published studies conducted outside of North America or Europe. Moreover, few were designed to compare different models of service delivery, and most relied on provider-reported information, rather than documenting hospital records or speaking to rape survivors themselves. ${ }^{21}$ This study used a pre/post-intervention design to test a nurse-driven model for strengthening post-rape services in a rural South African setting. The study had a number of strengths. A range of qualitative and quantitative methods were used to evaluate change, allowing for triangulation across these different methods. Thus, shifts in provider practice as measured in the chart review could be compared to corresponding changes observed through the patient interview. Objective measurement tools for quality of care indicators were developed based on standards of care outlined in national treatment guidelines, and measures of effect were adjusted for a range of potential confounders. Although it would have been ideal to have included a control group, for ethical and logistical reasons this was not feasible. It is difficult, therefore, to completely exclude the possibility that observed changes would anyway have occurred over time or that apparent intervention effects were not in part the result of confounding by other variables.

In terms of potential selection bias, the pre- and post- intervention populations sampled in the chart review and client interview were well matched, as were those eligible for the patient interview and those actually interviewed. It was not possible to assess for differences relating to socio-economic status, as this data is not routinely captured in hospital charts. Thus, it is possible that, in spite of offering a travel stipend, those who chose to come for the interview were better off than those who did not. It is also possible that they differed in terms of personal attributes (e.g. were more empowered or assertive). However, there is little reason to think that such differences would be differentially distributed between the pre- and post-intervention groups. Thus, while these factors might influence the generalizability of the findings, it is less likely that they would systematically bias the impacts observed. Similarly, although it is possible that patients may have been inclined to report favorably about services at the hospital, they did not know whether they were in the pre- or postintervention group, and therefore it is unlikely that this would have affected comparisons between these groups 


\section{CONCLUSIONS}

Taken together, the results of the Refentse study suggest that it is possible to improve comprehensive sexual assault services including PEP within a public sector hospital, using existing staff and resources, and that with additional training, nurses can play an expanded role in this care. Although further research, particularly in developing countries, is urgently needed, the study provides a number of important programmatic lessons regarding management of sexual assault in such settings (Figure 3).

The study also confirms the high levels of child sexual abuse reported in South Africa, with onequarter of cases in our study involving children younger than 14 years old, and half involving those younger than 18 years old. A particularly alarming finding is the age range captured in this study, where the youngest case involved an infant of 3 months and the oldest a 94 year-old grandmother. It is likely that hospital-based figures do not reflect the true magnitude and distribution of sexual violence in communities, and that those who are reluctant to press charges are also those least likely to seek healthcare. Few cases involving males were reported in this study - a warning that the needs of this particular population are likely not being met.

Remaining challenges include sustaining improvements at the study hospital and sharing program and policy lessons more widely. To this end, the program's monitoring and evaluation tools have been simplified and institutionalized within the OPD, and statistics on key indicators (e.g. provision of VCT and PEP) are now routinely reported to hospital management as well as district and provincial health managers responsible for sexual assault care and PEP. Lessons and tools from this study have been shared with provincial and national stakeholders within the South African Department of Health and Social Welfare, and training provided to over 800 health managers and service providers. Findings from the study have contributed to the development of the WHO/ILO Policy and Guidelines on Occupational and Non-Occupational HIV Post Exposure Prophylaxis ${ }^{61}$ and the Refentse model is now informing efforts to strengthen linkages within hospitals and with the judicial system in eight countries within East and Southern Africa. ${ }^{62}$ The management of STIs and unwanted pregnancy, and the provision of VCT and anti-retroviral drugs are not competencies specific to the care of women who have been raped. Our baseline assessment revealed significant institutional and provider gaps in these areas - findings that have also been noted nationally - and it is likely that such shortcomings reflect implications for the routine delivery of such services. ${ }^{6}$ Providing high quality care to survivors of sexual violence is a vital goal in its own right. Our research suggests that taking an institutional, nurse-driven approach to improving such care can simultaneously provide a critical opportunity to strengthen and integrate existing RH/HIV services a goal that may carry additional and far-reaching benefits. 
Figure 3: Emerging Lessons for Strengthening Sexual Assault Care in South Africa

1. Post rape care can be effectively integrated into existing HIV/RH services at the district hospital level in South Africa. Most diagnostic tests and treatments are generally available within the hospital; however, they may be scattered across different departments and service providers, leading to obstacles and delays in providing care.

2. In this context, introducing a hospital rape management policy may be important in establishing an institutional framework for coordinating care. Such a policy should lay out the responsibilities of a range of actors, beyond health care workers (e.g. clerks, pharmacy, laboratory, VCT counselors, social workers), as many of these providers impact directly on patient care. Such policies may also reduce the scope for individual providers to allow personal judgments and attitudes to shape their treatment of patients.

3. Specifying a designated room for treating cases of sexual assault may be useful for reducing delays, centralizing services and increasing privacy. Medications and diagnostic tests can be stored and dispensed directly from this room, minimizing delays and the need for additional providers.

4. Treatment protocols can systematize care and make it easier for providers to follow National Management Guidelines. Such protocols are particularly important given the lack of training received by many healthcare workers, and their high turnover within health facilities.

5. Most obstacles to providing PEP are institutional rather than patient-driven. From the patient's perspective, HIV infection is the main health concern, and most come to hospital within 72 hours. However, lack of VCT services at time of presentation may be a serious bottleneck, and because most patients present to hospital after-hours, VCT should be made available 24-hours a day.

6. Because of conventional practices for dispensing medications, PEP may be the last step in the hospital visit. Therefore providing a stat dose of PEP can reduce the time interval to first dose.

7. In rural areas, few patients are able to return to hospital after initial presentation. Therefore, wherever possible, all diagnostic tests and treatment should be provided on the first visit. For those eligible for PEP, a full 28-day course should be dispensed on the first visit. Same-day provision of anti-emetics and medication counseling are important for encouraging adherence.

8. Nurses can play a much greater role in the provision of post-rape care, and this may be particularly important in rural areas where there are few doctors. Further research and experience in training nurses to perform forensic exams is needed to guide policy and practice.

9. Working with other sectors is critical. Because the majority of rape survivors present to police first, cooperation with this sector can lessen delays to treatment, and provide an opportunity to strengthen medico-legal services. Similarly, in the absence of Rape Crisis Centers or other NGOs, ongoing counseling and support can be provided by strengthening referral systems to existing service providers, such as psychiatric nurses or social workers. 


\section{REFERENCES}

1. UNAIDS. AIDS epidemic update: December 2007. Geneva: UNAIDS, 2007.

2. Garcia-Moreno C, Watts C. Violence against women: It's importance for HIV/AIDS. AIDS 2000;14 (Suppl 3):S253-S265.

3. Rao Gupta G. How men's power over women fuels the HIV epidemic. BMJ. 2002;324:183-4.

4. UNAIDS. Report on the global HIV/AIDS epidemic. Geneva: UNAIDS, 2006.

5. Jewkes R, Abrahams N. The epidemiology of rape and sexual coercion in South Africa: an overview. Social Science and Medicine 2002;55 (7):1231-44.

6. Christofides NJ, et al. "Other patients are really in need of medical attention"- the quality of health services for rape survivors in South Africa. Bulletin of the World Health Organisation 2005; 83(7):495-502.

7. South African Police Services. Rape in the RSA for the financial years 1995/1995 to 2003/2004. Pretoria: South African Police Services, Crime Information Analysis Center, 2004.

8. Interpol. International Crime Statistics. Lyon: Interpol, 2004.

9. Crime Information Analysis Centre (CIAC). South African Police Service Crime Statistics. Pretoria, 2000.

10. World Health Organisation. Sexual Violence. World Report on Violence and Health. Geneva: World Health Organisation, 2002.

11. Dunkle K, Jewkes R, Brown H, Yoshinama M, Gray G, McIntyre J, et al. Prevalence and Patterns of Gender based Violence and Revictimization among Women Attending Antenatal Clinics in Soweto, South Africa 2004. American Journal of Epidemiology 2004;160(3):230-239.

12. Garcia-Moreno C, Jansen H, Ellsberg M, Heise L, Watts C. WHO Multi-country study on women's health and domestic violence against women: Initial results on prevalence, health outcomes, and women's responses. Geneva: World Health Organisation, 2005.

13. World Health Organisation. Guidelines for Medico-legal Care for Victims of Sexual Violence. Geneva, 2003.

14. Suffla S, Seedat M, Nascimento A. Evaluation of Medico-legal Services in Gauteng: Implications for the development of best practices in the after-care of rape survivors.: UNISA institute for Social and Health Sciences and Centre for Peace Action and MRC-Unisa Crime, Violence and Injury Lead Programme, United Nations Development, 2001.

15. Kerr E, Cottee C, Chowdhury R, Jawad R, Welch J. The Haven: A pilot referral centre in London for cases of serious sexual assault. BJOG: An International Journal of Obstetrics and Gynecology 2003;110:267-271. 
16. White BH, Kurpius SER. Attitudes toward rape victims, effects of gender and professional status. Journal of Interpersonal Violence 1999;14(9):989-995.

17. Bell CC, Jenkins EJ, Kpo W, Rhodes H. Response of emergency rooms to victims of interpersonal violence. Hospital and Community Psychiatry 1994;45(2):142-146.

18. Rosenberg KD, DeMunter JK, Liu J. Emergency contraception in emergency departments in Oregon, 2003. American Journal of Public Health 2005;95(8):1-5.

19. Lewis C, DiNitto D, Nelson TS, Just MM, Campbell-Ruggaard J. Evaluation of a rape protocol: A five year follow-up with nurse managers. Journal of the American Academy of Nurse Practitioners 2003;15(1):34-39.

20. Guedes A, Bott S, Cuca Y. Integrating systematic screening for gender-based violence into sexual and reproductive health services: Results of a baseline study by the International Planned Parenthood Federation, Western Hemisphere Region. International Journal of Gynecology and Obstetrics 2002;78(Suppl 1):S57-S63.

21. Martin SL, Young SK, Billings DL, Bross CC. Health Care-Based Interventions for Women Who Have Experienced Sexual Violence: A Review of the Literature. Trauma, Violence, and Abuse 2007;8(1):3-18.

22. Azikiwe N, Wright J, Cheng T, D'Angelo LJ. Management of rape victims (regarding STD treatment and pregnancy prevention): Do academic emergency departments practice what they preach? Journal of Adolescent Health 2005;36:446-448.

23. Myles JE, Hirozawa A, Katz, , M. H. K, R.,, Bamberger JD. Postexposure prophylaxis for HIV after sexual assault. Journal of the American Medical Association 2000;284(12):1516-1517.

24. Kim J, Martin L, Denny L. Rape and HIV Postexposure Prophylaxis: Addressing the Dual Epidemics in South Africa. Reproductive Health Matters 2003;11:101-12.

25. Roland ME. Postexposure prophylaxis after sexual exposure to HIV. Curr Opin Infect Dis 2007;20:39-46.

26. South African National Department of Health. Sexual Assault Policy. Pretoria, 2005.

27. South African National Department of Health. National Management Guidelines for Sexual Assault. Pretoria: South African National Department of Health, 2005.

28. Human Rights Watch. Deadly Delay: South Africa's Efforts to Prevent HIV in Survivors of Sexual Violence. Human Rights Watch Report 2004;16(3).

29. Christofides C, Webster N, Jewkes R, Penn-Kekana L, Martin L, Abrahams N, et al. T he State of Sexual Assault Services: Findings from a Situation Analysis of Services in South Africa. Pretoria: The South African Gender-based Violence and Health Initiative, 2003. 
30. Vetten L, Haffejee SS. Factors affecting Adherence to Post-Exposure Prophylaxis in the Aftermath of Sexual Assault: Key Findings From Seven Sites in Gauteng Province. Report prepared for the Gauteng Department of Health. Pretoria, 2005.

31. Askew, I. and Berer, M. The Contribution of Sexual and Reproductive Health Services to the Fight Against HIV/AIDS: A Review. Reproductive Health Matters 2003;11(22):51-73.

32. Population Council Frontiers in Reproductive Health Program and RADAR SoPH, University of the Witwatersrand. Frontiers News: Integrating post-rape care services into existing reproductive health programs in South Africa. Johannesburg: Population Council, 2005:6-7.

33. World Health Organisation. Putting women first: Ethical and safety recommendations for research on domestic violence against women. WHO/EIP/GPE/99.2. Geneva: WHO, 2001.

34. Ellsberg M, Heise L. Researching Violence Against Women: A Practical Guide for Researchers and Activists. Washington DC: World Health Organization, PATH, 2005.

35. Barros AJD, Hirakata VN. Alternatives for logistic regression in cross-sectional studies: an empirical comparison of models that directly estimate the prevalence ratio. BMC Medical Research Methodology 2003;3(21):http://www.biomedcentral.com/1471-2288/3/21.

36. Coast J. Is economic evaluation in touch with society's health values? BMJ. 2004;329(7476):12336.

37. Ellis J C, Ahmad S, Molyneux E M. Introduction of HIV post-exposure prophylaxis for sexually abused children in Malawi. Arch. Dis. Child 2005;90:1297-1299.

38. Christofides NJ, Muirhead D, Jewkes RK, et. al. Women's experiences of and preferences for services after rape in South Africa: an interview study. BMJ 2006;332:209-213.

39. Speight CG, Klufio A, Kilonzo SN, Mbugua C, Kuria E, Bunn JE, et al. Piloting post-exposure prophylaxis in Kenya raises specific concerns for the management of childhood rape. Transactions of the Royal Society of Tropical Medicine and Hygiene 2006;100:14-18.

40. Carries S, Muller F, Muller FJ., Morroni C, Wilson D. Characteristics, Treatment, and Antiretroviral Prophylaxis Adherence of South African Rape Survivors. I Acquir Immune Defic Syndr 2007;46:68-71.

41. HIV PEP uptake among sexual assault survivors: results of an observational study. Poster discussion: 4th IAS Conference on HIV Pathogenesis, Treatment and Prevention, Abstract no. MOPDC04; 2007; Sydney, Australia.

42. Garcia MT, Fiqueiredo RM, Moretti ML, Resende MR, Bedoni AJ, Papaiordanou PMO. Postexposure prophylaxis after sexual assaults: A prospective cohort study. Sexually Transmitted Diseases 2005;32(4):214-219. 
43. Linden JA, Oldeg P, Mehta SD, McCabe KK, LaBelle C. HIV postexposure prophylaxis in sexual assault: Current practice and patience adherence to treatment recommendations in a large urban teaching hospital. Academic Emergency Medicine 2005;12(7):640-646.

44. Wiebe ER, Comay SE, McGregor M, Ducceschi S. Offering HIV prophylaxis to people who have been sexually assaulted: 15 months' experience in a sexual assault service. Canadian Medical Association Journal of Acquired Immuno Deficiency Syndrome 2000;162(5): 641-645.

45. Sonder G, Regez RM, et. al. Trends in HIV-PEP prescription and compliance after sexual exposure in Amsterdam, 2000-2004. Sex Transm Dis 2007;34:288 -293.

46. Limb S, Kawsar M, Forster G E. HIV post-exposure prophylaxis after sexual assault: the experience of a sexual assault service in London. International Journal of STD \& AIDS 2002;13.

47. Neu N, Heffernan-Vacca S, Millery M, Stimell M, Brown J. Postexposure Prophylaxis for HIV in Children and Adolescents After Sexual Assault: A Prospective Observational Study in an Urban Medical Center. Sexually Transmitted Diseases 2007;34(2):65-68.

48. Kahn JO MJ, Roland ME, al. e. Feasibility of postexposure prophylaxis (PEP) against human immunodeficiency virus infection after sexual or injection drug use exposure: the San Francisco PEP Study. Journal of Infectious Diseases 2001;183:707-14.

49. Gibb AM, McManus T, Forster GE. Should we offer antibiotic prophylaxis post sexual assault? International Journal of STD and AIDS 2003;14:99-102.

50. Roland ME. A Model for Communitywide Delivery of Postexposure Prophylaxis After Potential Sexual Exposure to HIV. Sexually Transmitted Diseases 2007;34(5):294-296.

51. Hachey M, van As AB. HIV postexposure prophylaxis in victims of child sexual abuse. Ann Emerg Med 2005;46:97-98.

52. Gerberding JL. Management of occupational exposures to blood-borne viruses. New England Journal of Medicine 1995;332:444-51.

53. Niu MT, et. al. Primary HIV type-1 infection: review of pathogenesis and early treatment intervention in human and animal retrovirus infections. Journal of Infectious Diseases 1993;168:1490-501.

54. Green WM, Panacek EA. Sexual assault forensic examinations in evolution. Journal of Emergency Medicine 2003;25:97-9.

55. Ledray LE, Simmelink K. Efficacy of SANE Evidence Collection: a Minnesota Study. Journal of emergency Nursing 1997;23:75-7.

56. Derhammer F, Lucente V, Reed JF, Young MJ. Using a SANE interdisciplinary approach to care of sexual assault victims. Journal on Quality Improvement 2000;26(8):488-495. 
57. Cleary SM, McIntyre D, Boulle A. The cost-effectiveness of Antiretroviral Treatment in Khayelitsha, South Africa - a primary data analysis. Cost Effectiveness and Resource Allocation 2006;4(20).

58. Cleary S. Equity and efficiency in health and health care for HIV-positive adults in South Africa [PhD Thesis]. Faculty of Health Sciences, University of Cape Town, 2007.

59. Supporting Translation of Evidence into Policy and Practice (STEPP). Assessment of the effectiveness of emergency contraception. Survivors of rape and sexual assault : policy and standardized management guidelines (H17/2004): http://www.mrc.ac.za/cochrane/emergency.pdf (accessed 16 January 2008), 2005.

60. Geffen N, Nattrass N, Raubenheimer C. The cost of HIV Prevention and Treatment in South Africa. CSSR Working paper No. 28.: Centre for Social Science Research, University of Cape Town, 2003.

61. ILO/WHO. Occupational and Non-occupational Post-exposure Prophylaxis for HIV Infection (HIV-PEP) Joint ILO/WHO Technical Meeting for the Development of Policy and Guidelines: Summary Report. Geneva: Institution of Labor Organisation and the World Health Organisation, 2005

62. The Population Council. Sexual and Gender-based Violence in East and Southern Africa, 2007. http://www.popcouncil.org/projects/RH ESASexGenderBasedViolence.html. 


\section{Population Council}

One Dag Hammarskjold Plaza, New York

New York NY

USA 10017

Tel: +12123390500

Fax: +12127556052

pubinfo@popcouncil.org

www.popcouncil.org 